\title{
Intraspecies comparison of Streptomyces pratensis genomes reveals high levels of recombination and gene conservation between strains of disparate geographic origin
}

James R Doroghazi ${ }^{1}$ and Daniel H Buckley ${ }^{2^{*}}$

\begin{abstract}
Background: Streptomyces are widespread bacteria that contribute to the terrestrial carbon cycle and produce the majority of clinically useful antibiotics. While interspecific genomic diversity has been investigated among Streptomyces, information is lacking on intraspecific genomic diversity. Streptomyces pratensis has high rates of homologous recombination but the impact of such gene exchange on genome evolution and the evolution of natural product gene clusters remains uncharacterized.

Results: We report draft genome sequences of four S. pratensis strains and compare to the complete genome of Streptomyces flavogriseus IAF-45-CD (=ATCC 33331), a strain recently reclassified to S. pratensis. Despite disparate geographic origins, the genomes are highly similar with $85.9 \%$ of genes present in the core genome and conservation of all natural product gene clusters. Natural products include a novel combination of carbapenem and beta-lactamase inhibitor gene clusters. While high intraspecies recombination rates abolish the phylogenetic signal across the genome, intraspecies recombination is suppressed in two genomic regions. The first region is centered on an insertion/deletion polymorphism and the second on a hybrid NRPS-PKS gene. Finally, two gene families accounted for over $25 \%$ of the divergent genes in the core genome. The first includes homologs of bldB (required for spore development and antibiotic production) while the second includes homologs of an uncharacterized protein with a helix-turn-helix motif ( $h p b$ ). Genes from these families co-occur with fifteen pairs spread across the genome. These genes have evidence for co-evolution of co-localized pairs, supporting previous assertions that these genes may function akin to a toxin-antitoxin system.
\end{abstract}

Conclusions: S. pratensis genomes are highly similar with exceptional levels of recombination which erase phylogenetic signal among strains of the species. This species has a large core genome and variable terminal regions that are smaller than those found in interspecies comparisons. There is no geographic differentiation between these strains, but there is evidence for local linkage disequilibrium affecting two genomic regions. We have also shown further observational evidence that the DUF397-HTH (bldB and hpb) are a novel toxin-antitoxin pair.

Keywords: Streptomyces, Comparative genomics, Bioprospecting, Homologous recombination, Genome evolution, Core genome, Pan-genome

\footnotetext{
* Correspondence: dbuckley@cornell.edu

${ }^{2}$ Department of Crop and Soil Sciences, Cornell University, Ithaca, USA

Full list of author information is available at the end of the article
} 


\section{Background}

Streptomyces are ubiquitous bacteria with many uncommon features and important industrial uses. They produce over half of the clinically useful antibiotics and a host of other bioactive, pharmaceutically relevant compounds [1]. The name Streptomyces means twisted fungus, reflecting the morphological and life cycle traits that these bacteria share with fungi. While Streptomyces taxonomy is notably problematic, multi-locus sequence analysis (MLSA) approaches are helping to resolve species boundaries in the genus [2-4]. For example, measurements of MLSA divergence in relation to DNA-DNA hybridization (DDH) values indicate that $0.7-$ $0.8 \%$ divergence of MLSA loci roughly delineates species boundaries in Streptomyces $[3,5,6]$. Though asexual, Streptomyces are capable of genetic exchange within and between species [7], and there is evidence of widespread horizontal gene transfer within and between species of the genus [8]. The implications of horizontal gene transfer for genome evolution within Streptomyces remains poorly described.

Streptomyces genomes deviate from those of other bacteria in several ways. There can be multiple genomes per cellular compartment, though asexual spores have a single genome copy. Hyphae elongate at the tip and form septa that define cellular compartments at regular intervals $[9,10]$. Roughly ten to twelve genomes can coexist inside of a single compartment $[7,11]$. Streptomyces genomes are linear and replicate from a bidirectional central ori, although they can exist as unstable, circular molecules [12-14]. The ends of their chromosomes consist of terminal inverted repeats (TIRs), and the length of these TIR regions can vary largely, from 167 bp to 1 $\mathrm{Mb}[15,16]$. Their plasmids can be circular or linear, and can mobilize chromosomal markers at high frequency during interspecies transfer [17]. The chromosome has been classified into two sections: the central, conserved core region and the more variable terminal chromosome arms [18].

One surprise revealed by genome sequencing of Streptomyces species is the presence of numerous cryptic secondary metabolite gene clusters $[19,20]$. These cryptic gene clusters encode products that are either silent or not identified as natural products during growth in the laboratory. For example, four decades of genetic analysis had identified four secondary metabolite gene clusters in the model organism Streptomyces coelicolor, but the first genome sequence revealed a total of 22 secondary metabolite gene clusters [19]. The diversity of natural product gene clusters is very high in Streptomyces compared to other bacterial genera. There is very little overlap in terms of shared natural product biosynthetic gene clusters between the currently closed Streptomyces genomes, all of which are from different species [21]. The intraspecies variability of natural product biosynthetic genes has not yet been determined through comparative genomic analysis of Streptomyces species. However, recent studies of Salinispora, another actinomycete genus rich in natural product biosynthetic genes, show high overall conservation within species [22].

The species $S$. pratensis has been described recently [23] to include isolates from a wide region of North America spanning sites found in North Carolina, New York, Michigan, and Quebec [8]. Nucleotide divergence of MLSA loci from strains of $S$. pratensis did not exceed $0.4 \%$, justifying their inclusion in a single species [8]. Very high levels of homologous recombination were detected in $S$. pratensis, sufficient to promote linkage equilibrium for alleles at MLSA loci [8]. Interspecies recombination is widespread among Streptomyces, although interspecies gene exchange occurs at a much lower rate than intraspecies gene exchange [8]. The genetic coherence of Streptomyces species is surprising given the potential for widespread gene exchange, the high level of nucleotide similarity between many different species, and the null expectations for highly recombining populations $[24,25]$. The maintenance of coherent genetic clusters that correspond to Streptomyces species suggests some mechanism for constraining interspecies gene exchange.

We have sequenced the genomes of four strains of the newly described species $S$. pratensis, including the type strain Ch $24^{\mathrm{T}}\left(=\mathrm{NRRL} \mathrm{B}-24916^{\mathrm{T}}\right)$. The four strains were isolated from edaphically similar sites separated by 740 $\mathrm{km}$. These draft genome sequences were compared with the complete genome of $S$. flavogriseus IAF-45-CD (=ATCC 33331), which has been reassigned recently to S. pratensis [23].

\section{Methods}

\section{Source of strains}

S. pratensis strain IAF-45-CD (=ATCC $33331=S$. flavogriseus strain IAF-45-CD) was sourced directly from ATCC. $S$. pratensis IAF-45-CD was isolated from compost in Laval, Canada [26]. The other four strains of S. pratensis were isolated directly from soil. Strains Will23 and Will26 were both isolated from Willsboro, NY N 44.38, W -73.38. Strains Ch2 and $\mathrm{Ch} 24^{\mathrm{T}}=\left(\mathrm{NRRL} B-24916^{\mathrm{T}}\right)$ were both isolated from Charlotte, NC (N 38.81, W -78.26), which is $740 \mathrm{~km}$ from the Willsboro site. Both sites are grassy fields which are edaphically similar. Isolation was carried out on glycerolarginine media [27], including cycloheximide (300 $\mathrm{mg} \mathrm{L}^{-1}$ ) and Rose Bengal (35 $\mathrm{mg} \mathrm{L}^{-1}$ ) as described previously [28]. Classification of these strains as $S$. pratensis has been described previously.

\section{DNA preparation and sequencing}

DNA was isolated as described previously [8]. DNA over 10 $\mathrm{kb}$ was selected using gel purification (MO-BIO, Carlsbad, CA) and submitted to the Cornell Life Sciences Core Laboratories Center for DNA sequencing. DNA libraries were prepared including MID tags and all four libraries were 
run together using GS FLX Titanium series reagents on a GS FLX instrument. Average read length for each isolate ranged from 422 to $425 \mathrm{bp}$. Additional summary genome statistics are given in Table 1. A de novo assembly was created with gsAssembler and a mapping assembly was created with gsMapper using the IAF-45-CD genome as reference. A final assembly was performed with gsAssembler using a combination of the original reads and faux reads created by breaking contigs from the independent gsAssembler and gsMapper runs into 1999 bp pieces with 50 bp overlap. Contigs were ordered using Mauve version 2.3.1 [29] and gene prediction was performed with Prodigal version 2.50 [30]. Genome sequences are available through Genbank BioProjects PRJNA252632-5.

\section{Genome analyses}

Mauve was used for genome alignment and to find positional orthologs and SNPs. The nucleotide sequences of core positional orthologs were aligned using ClustalW version 1.83 [31]. Distances were calculated with DNAdist in the PHYLIP package version 3.69 [32]. Annotation of divergent core genes was performed using Reverse Position Specific BLAST 2.2.25+ against the Conserved Domain Database (CDD.v2.32) [33,34]. GO term enrichment was performed using topGO version 2.10.0 [35] within Bioconductor (Biobase version 2.18.0) [36]. Secondary metabolite biosynthetic gene clusters were found with AntiSMASH version 1.1.0 [37]. BldB and $\mathrm{Hpb}$ amino acid sequences were aligned using ClustalW version 1.83 with default alignment parameters [31]. The maximum likelihood trees were created using FastTreeMP version 2.1.5 [38]. Tree visualization was performed with the Python library ETE version 2.2 [39].

The other genomes used for the analyses of phylogenetic signal are: Helicobacter pylori F32 (NC_017366.1), $H$. pylori F57 (NC_017367.1), H. pylori F16 (NC_017368.1), H. pylori 51 (NC_017382.1), H. pylori F30 (NC_017365.1), Mycobacterium tuberculosis str. Erdman = ATCC 35801 (AP012340.1), M. tuberculosis KZN 4207 (NC_016768.1), M. tuberculosis RGTB423 (NC_017528.1), M. tuberculosis CTRI-2 (NC_017524.1), and M. tuberculosis CCDC5079 (NC_017523.1). All analyses not described above were performed using custom Perl scripts. Significance testing for regions of extended linkage was performed using random draws to determine compatible sites based on the exponential decay discussed in the text across $20 \mathrm{~kb}$ tracts of the genome centered on every SNP and repeated 100 times.

\section{Results}

\section{Genome summary statistics}

The draft genomes for the $S$. pratensis strains (including plasmids) range from 7510568-7623889 bp (for contigs over $2000 \mathrm{bp}$ ) and have 6723 to 6782 predicted genes and an average $\mathrm{G}+\mathrm{C}$ content of $71 \%$ (Table 1). The draft genomes do not allow for conclusive delineation between chromosomal and plasmid DNA due to the presence of linear plasmids. Increasing coverage (from $16 x$ to $27 x$ ) corresponds to an increase in N50 from 128423 to 171112 and a decrease in the number of contigs (from 128 to 87 ) for each assembly; there is no relationship between coverage and assembled genome size.

\section{Conservation of gene content}

To determine the similarity of gene content in the five genomes, we determined orthologous genes based on position and similarity in whole genome alignments calculated with Mauve. A surprising 5773 positional orthologs are shared by all five strains indicating that the core genome comprises $85-88 \%$ of gene content for the five genomes. Adding additional genomes beyond the second genome causes only a modest reduction in the size of the core genome (Figure 1B). There is a clear pattern that most genes are either unique to one strain $(2,407$ genes total, ranging from 449 to 523 unique genes per strain) or shared by all five strains (5773 genes) (Figures 1A and 1C). This pattern is established from all pairwise comparisons of genomes rather than comparison solely to IAF-45-CD, and thus is not likely to result from differences in coverage between complete and draft genomes. It is expected that the number of genes unique to one strain will be dependent on the total number of genomes sampled and hence the proportion of unique genes may decrease even further as more genomes of the species are sampled.

Variable genes are not randomly distributed across the chromosome (Figure 1D). There are 11 genomic islands of $10 \mathrm{kbp}$ or longer in IAF-45-CD that are enriched

Table 1 Genome assembly summary statistics

\begin{tabular}{lccccccc}
\hline Strain & Assembled size (bp) & Contigs & N50 (bp) & ORFs & Reads & Coverage & G + C (\%) \\
\hline Will23 & $7517207(7521185)$ & $97(112)$ & 171112 & 6723 & 357319 & $20.0 \times$ & 71.1 \\
Will26 & $7510568(7516455)$ & $87(110)$ & 176481 & 6739 & 492343 & $27.9 \times$ & 71.1 \\
Ch2 & $7538829(7544224)$ & $128(149)$ & 128423 & 6782 & 284409 & $16.0 x$ & 71.1 \\
Ch24 & $7623889(7630757)$ & $110(137)$ & 132873 & 6770 & 332240 & $18.5 \times$ & 71.0 \\
IAF-45-CD (pSFLA01, pSFLA02) & $7337497(188552,130055)$ & $1(1,1)$ & NA & $6443(201,126)$ & NA & NA & $71.1(67.8,67.2)$ \\
\hline
\end{tabular}

Data is provided both for large contigs (>2000 bp) and for all contigs (provided parenthetically). For IAF-45-CD, the two numbers in parentheses indicate data for the plasmids pSFLA01 and PSFLA02 respectively. 


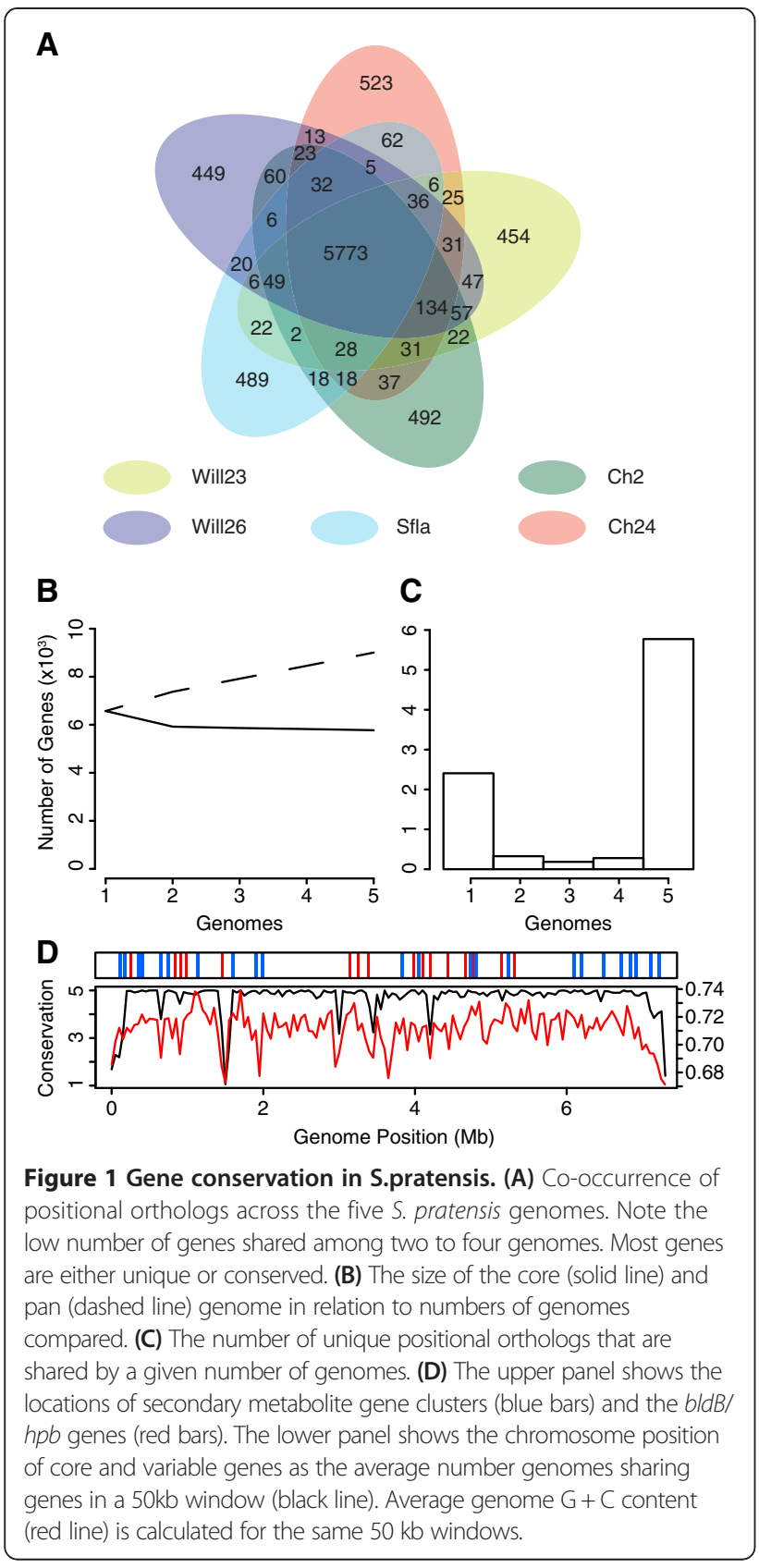

( $\geq 50 \%$ of gene content) in genes specific to IAF- $45-\mathrm{CD}$ (Figure 1D). These islands account for 376505 bp of the genome, or $5.1 \%$ of the chromosome. The terminal regions of the chromosome are among the regions enriched in variable genes (Figure 1D), supporting the hypothesis that genome variability in Streptomyces increases at chromosomal termini. The terminal variable regions each comprise 112 $\mathrm{kbp}$ and $36 \mathrm{kbp}$ of the chromosome, based on these criteria. Certain GO terms are overrepresented among the strain specific genes (Table 2 lists genes unique to IAF-45-CD). Primary among them are genes involved in transposition and integration, as well as metabolic processes. The largest variable region unique to IAF-45-CD is a putative mobile element likely to be involved in phage defense (Figure 2). This region, which lies between Sfla_1274 and Sfla_1300, contains multiple DNA methyltransferases, nucleases and a predicted gene for an abortive phage infection protein. This region also encodes putative transfer genes that are highly similar to a genomic neighborhood found in "S. coelicolor" A3(2) M145. Taken altogether, these results show that there is a highly similar overall coding capacity between these five intraspecific strains, and that the variability that does exist is isolated to chromosome termini and genomic islands likely to have been acquired through horizontal gene transfer.

To determine the variation in natural product biosynthetic gene clusters between these five strains, we used positional orthologs as described above to determine the presence/absence of gene clusters detected using the program antiSMASH. All five strains have 100\% conservation of putative secondary metabolic biosynthetic gene clusters in terms of presence (not sequence identity). S. pratensis contains a novel combination of biosynthetic gene clusters including one that synthesizes a beta-lactam antibiotic and another that synthesizes a beta-lactamase inhibitor (Figure 3). A similar combination of beta-lactam and betalactamase inhibitor gene clusters is present in S. clavuligerus ATCC 27064, and in fact the latter gene cluster in S. pratensis most closely resembles a gene cluster in S. clavuligerus ATCC 27064 which is responsible for production of the beta-lactamase inhibitor clavulanic acid. In contrast, the beta-lactam gene cluster in S. clavuligerus ATCC 27064 produces cephamycin and is distantly related to the betalactam gene cluster found in $S$. pratentis. The $S$. pratensis beta-lactam gene cluster most closely resembles that reported for MM 4550 from S. argenteolus ATCC 11009 [40]. MM 4550 is within the carbapenem class of beta-lactams [41], but is distantly related to the cephamycin gene cluster (e.g. the beta-lactam synthases from $S$. clavuligerus and $S$. pratensis share only $32 \%$ identity over $81 \%$ of $\mathrm{cmmM}$ ). In addition, biosynthetic gene clusters in Streptomyces have been shown to associate only on short evolutionary time scales [42], suggesting that these two sets of gene clusters have different evolutionary origins in S. pratensis and S clavuligerus. Taken together, this shows that the pharmaceutically important association of these two antibiotic classes, a betalactam and a beta-lactamase (antibiotic resistance) inhibitor, has evolved independently on more than one occasion.

\section{Sequence level conservation}

Genome-wide intraspecific sequence divergence was calculated from whole the genome alignment indicating a high level of sequence similarity. Core gene sequences from the four draft genomes had a median nucleotide dissimilarity to IAF-45-CD of 0.0041 (s.d. = 0.016, Figure 4A), or 99.59\% nucleotide identity. The median density of SNPs calculated 
Table 2 GO term enrichment in genes unique to IAF-45-CD

\begin{tabular}{|c|c|c|c|c|c|}
\hline GO ID & Term & All & Unique & Expected & $p$-value \\
\hline GO:0006259 & DNA metabolic process & 92 & 22 & 2.39 & $8.12 \mathrm{E}-14$ \\
\hline GO:0015074 & DNA integration & 11 & 10 & 0.29 & $7.02 \mathrm{E}-13$ \\
\hline GO:0003676 & nucleic acid binding & 439 & 34 & 11.29 & $3.40 \mathrm{E}-07$ \\
\hline GO:0044260 & cellular macromolecule metabolic process & 700 & 42 & 18.2 & $8.12 \mathrm{E}-07$ \\
\hline GO:0090304 & nucleic acid metabolic process & 555 & 37 & 14.43 & 1.09E-06 \\
\hline GO:0003677 & DNA binding & 356 & 29 & 9.16 & $3.47 \mathrm{E}-06$ \\
\hline GO:0034641 & cellular nitrogen compound metabolic pro... & 699 & 41 & 18.18 & 3.47E-06 \\
\hline GO:0006310 & DNA recombination & 18 & 8 & 0.47 & 4.47E-06 \\
\hline GO:0006139 & nucleobase-containing compound metabolic... & 631 & 38 & 16.41 & $1.09 \mathrm{E}-05$ \\
\hline GO:0006807 & nitrogen compound metabolic process & 725 & 41 & 18.85 & $1.19 \mathrm{E}-05$ \\
\hline GO:0043170 & macromolecule metabolic process & 803 & 42 & 20.88 & 7.57E-05 \\
\hline GO:0006304 & DNA modification & 7 & 5 & 0.18 & $1.92 \mathrm{E}-04$ \\
\hline GO:0004803 & transposase activity & 4 & 4 & 0.1 & 3.03E-04 \\
\hline GO:0006305 & DNA alkylation & 4 & 4 & 0.1 & 3.83E-04 \\
\hline GO:0006306 & DNA methylation & 4 & 4 & 0.1 & 3.83E-04 \\
\hline GO:0006313 & transposition, DNA-mediated & 4 & 4 & 0.1 & 3.83E-04 \\
\hline GO:0032196 & transposition & 4 & 4 & 0.1 & 3.83E-04 \\
\hline GO:0040029 & regulation of gene expression, epigeneti... & 4 & 4 & 0.1 & 3.83E-04 \\
\hline GO:0044237 & cellular metabolic process & 1096 & 48 & 28.5 & 1.09E-03 \\
\hline GO:0008152 & metabolic process & 2048 & 66 & 53.26 & $8.94 \mathrm{E}-03$ \\
\hline GO:0043414 & macromolecule methylation & 7 & 4 & 0.18 & $1.28 \mathrm{E}-02$ \\
\hline GO:0043412 & macromolecule modification & 63 & 9 & 1.64 & $2.28 \mathrm{E}-02$ \\
\hline GO:0032259 & methylation & 16 & 5 & 0.42 & $3.28 \mathrm{E}-02$ \\
\hline
\end{tabular}

Gene ontology (GO) classifications were used to determine whether certain functional categories are enriched in the pool of genes unique to IAF-45-CD. The category 'All' indicates annotations for the entire IAF-45-CD chromosome; 'Unique' indicates annotations for genes unique to IAF-45-CD; 'Expected' indicates the number of unique genes expected if sampled at random from the pool of all unique genes found in the five genomes. The Bonferroni corrected $p$-values indicate the probability that the observed unique gene frequency is due to chance.

in $50 \mathrm{~kb}$ windows across the conserved regions of all five genomes is 0.009 (s.d. $=0.010$, Figure 4B).

Core genes with more than $5 \%$ divergence to a positional ortholog in IAF-45-CD were identified and investigated. There are 59 such genes present in 33 regions across the genome (Table 3). These regions were delimited based on whether the identified genes co-occurred within $10 \mathrm{kbp}$. The 33 regions contained a median of 2 divergent genes and varied in length from 156 to $15570 \mathrm{bp}$. Two of these divergent regions of the core genome are worth noting.

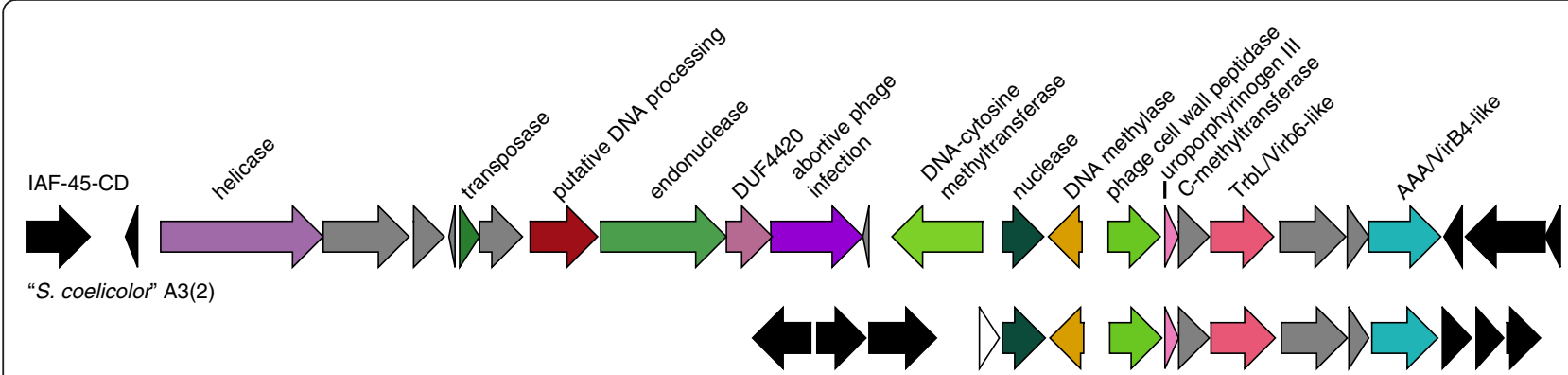

Figure 2 A putative mobile element unique to IAF-45-CD. A subset of genes from the large unique genomic island found at the $1.5 \mathrm{Mbp}$ locus of IAF-45-CD. A region with identical gene content and high similarity (e.g. 99\% predicted TrbL amino acid sequence identity) was found in the genome of "S. coelicolor" A3(2). For comparison, the rpoB and gyrB amino acid sequences of IAF-45-CD and A3(2) are 96\% and 87\% identical, respectively. Genes that have a putative functional assignment are shown in color, genes outside of the region of interest are shown in black, and genes with no putative functional assignment are shown in gray. Orthologous genes are shown in the same color. 


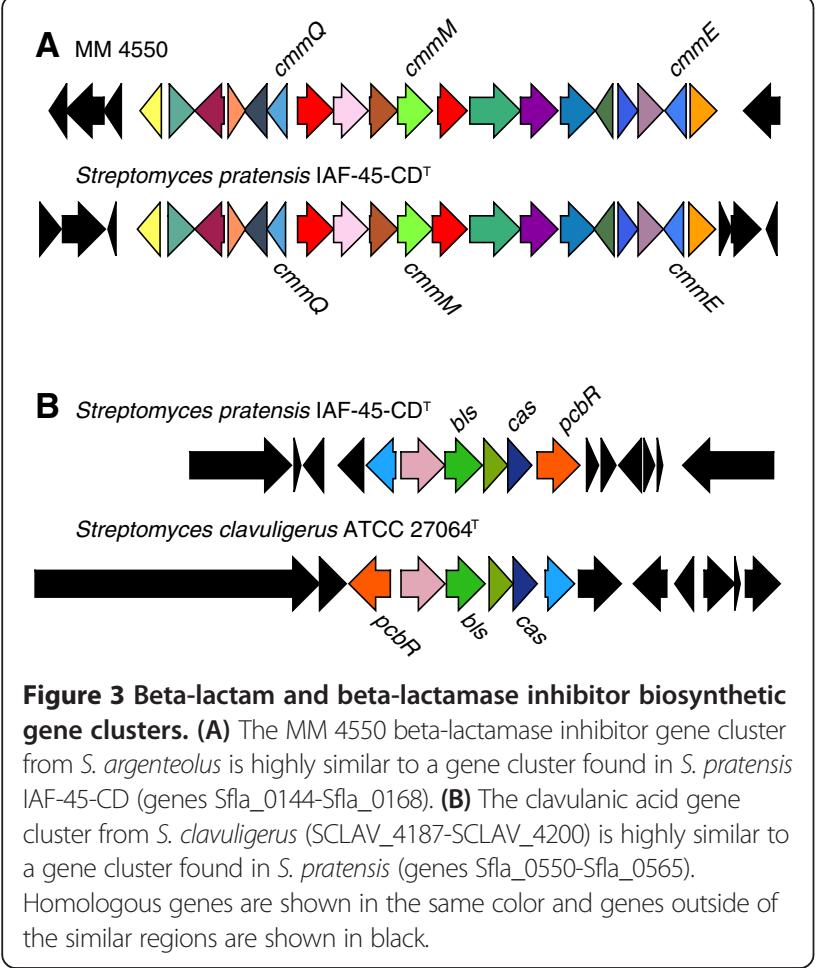

Sfla_4158 is predicted to encode a 51 amino acid lanthipeptide precursor in a putative two-component lanthipeptide biosynthetic gene cluster. The $\mathrm{N}$-terminal 29 amino acids are identical across all S. pratensis genomes, but there are two indels and 11 point mutations resulting in six amino acid substitutions among the 22 amino acids at the C-terminal of Sfla_4158. As lanthipeptide precursors are post-translationally modified to form the final product, these amino acid changes must impact directly the final structure of this lanthipeptide. In the second region of interest are found Sfla_1620 and 1621 and 1627, which are annotated respectively as a peptidase, a hypothetical protein, and a gene required for antibiotic production and formation of aerial hyphae (bldB). Sfla_1620 is most similar to the type VII secretion-associated serine protease mycosin and Sfla_1621 is most similar (detected with DELTA-BLAST [43]) to ESX-1 secretion-associated alanine and proline rich proteins involved in the ESX-1 type VII secretion system in Mycobacterium. In S. pratensis these genes occur as part of an operon comprised of several genes that have orthologs required for morphogenetic development and spore formation in "S. coelicolor" A3(2) and which are regulated in part by BldB (Sfla_1627).

A total of 19 of the 59 divergent genes described above were found to belong to one of two gene families. One of these is putatively the transcriptional regulator bldB. Each genome contained 18 genes with homology to bldB. Nucleotide and amino acid alignments of bldB orthologs have a mean proportion of 0.118 (s.d. $=0.121$ )

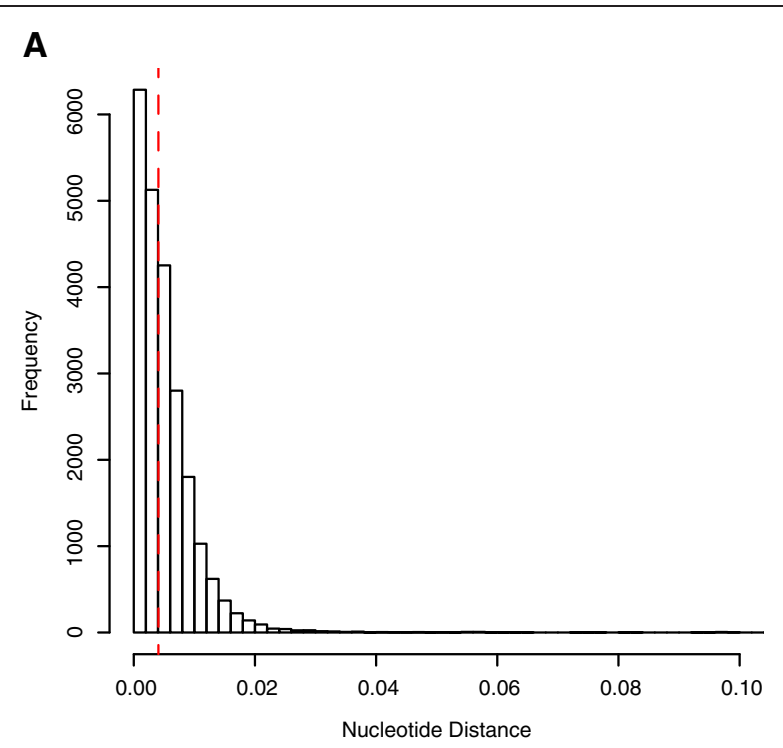

B

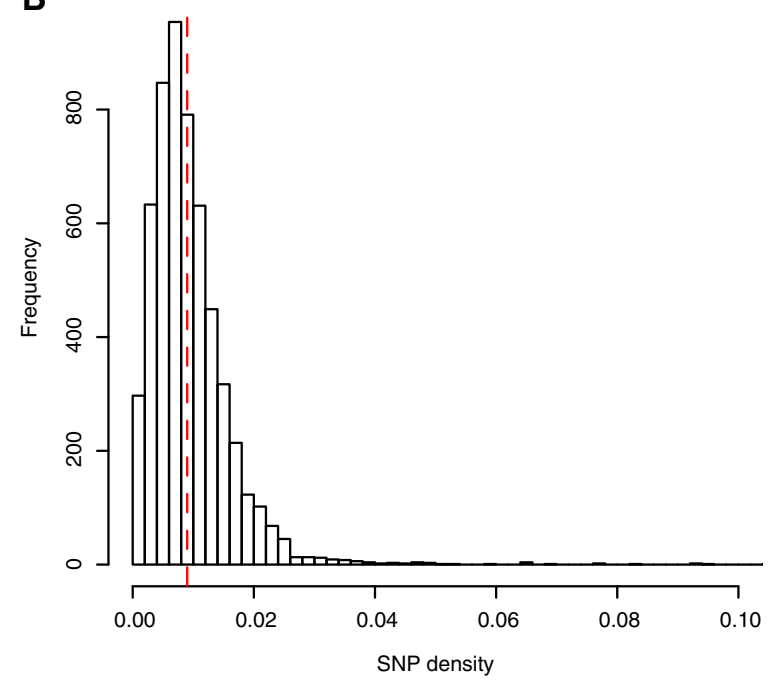

Figure 4 Sequence conservation. (A) Pairwise genetic distances in conserved genes between the four draft genomes and IAF-45-CD. Only genes conserved in all five genomes were used. The median pairwise distance, 0.0041 , is shown with a dashed red line. (B) SNP density sampled in $50 \mathrm{~kb}$ windows across the genome. The median SNP density, 0.009 , is shown with a dashed red line.

and 0.155 (s.d. $=0.142$ ) variable sites, respectively. The other transcriptional regulator, which we refer to as helixturn-helix partner of BldB $(h p b)$, contains an N-terminal helix-turn-helix motif, but is otherwise as yet uncharacterized. Each genome contains $16 \mathrm{hpb}$ homologs, and nucleotide and amino acid alignments of $h p b$ orthologs have a mean proportion of 0.188 (s.d. $=0.126$ ) and 0.208 $($ s.d. $=0.143)$ variable sites, respectively. We observe that genes of these families are typically adjacent or occur together as an operon. Only 2 of the $18 \mathrm{bldB}$ homologs lack an $h p b$ partner and only 1 of the $16 \mathrm{hpb}$ homologs lacked a bldB partner. One putative operon (Sfla_2921-2923) 
Table 3 Highly divergent core genes

\begin{tabular}{|c|c|c|}
\hline Gene name & CD hit & Predicted function \\
\hline Sfla_0019 & N/A & hypothetical \\
\hline Sfla_0256 & pfam05120 & Gas vesicle protein $G$ \\
\hline Sfla_0695 & TIGR03062 & Unknown, required for phage infection \\
\hline Sfla_0790 & pfam04149 & BldB family \\
\hline Sfla_0791 & N/A & $\mathrm{HBP}$ \\
\hline Sfla_0852 & pfam04149 & BldB family \\
\hline Sfla_0853 & N/A & $\mathrm{HBP}$ \\
\hline Sfla_1620 & TIGR03921 & $\begin{array}{l}\text { type VII secretion-associated } \\
\text { serine protease mycosin }\end{array}$ \\
\hline Sfla_1621 & N/A & hypothetical \\
\hline Sfla_1909 & N/A & hypothetical \\
\hline Sfla_1910 & N/A & hypothetical \\
\hline Sfla_1911 & N/A & cholesterol esterase \\
\hline Sfla_2255 & N/A & hypothetical \\
\hline Sfla_2258 & N/A & hypothetical \\
\hline Sfla_2259 & N/A & hypothetical \\
\hline Sfla_2260 & N/A & hypothetical \\
\hline Sfla_2267 & N/A & TadE-family protein \\
\hline Sfla_2678 & pfam04149 & BldB family \\
\hline Sfla_2704 & pfam07228 & stage II sporulation E protein \\
\hline Sfla_2785 & smart00530 & $\mathrm{HBP}$ \\
\hline Sfla_2786 & pfam04149 & BldB family \\
\hline Sfla_2892 & COG0642 & Signal transduction histidine kinase \\
\hline Sfla_2916 & pfam06259 & alpha/beta hydrolase fold \\
\hline Sfla_2921 & N/A & $\mathrm{HBP}$ \\
\hline Sfla_2922 & pfam04149 & BldB family \\
\hline Sfla_2930 & N/A & hypothetical \\
\hline Sfla_2931 & N/A & hypothetical \\
\hline Sfla_3203 & TIGR02614 & $\begin{array}{l}\text { FtsW - Biosynthesis and degradation } \\
\text { of murein sacculus and peptidoglycan }\end{array}$ \\
\hline Sfla_3555 & pfam04149 & BldB family \\
\hline Sfla_3556 & N/A & $\mathrm{HBP}$ \\
\hline Sfla_3613 & COG4188 & dienelactone hydrolase \\
\hline Sfla_3767 & cd06260 & domain of unknown function \\
\hline Sfla_3776 & N/A & hypothetical \\
\hline Sfla_3819 & N/A & hypothetical \\
\hline Sfla_3854 & N/A & $\mathrm{HBP}$ \\
\hline Sfla_3855 & pfam04149 & BldB family \\
\hline Sfla_3958 & N/A & hypothetical \\
\hline Sfla_4039 & N/A & hypothetical \\
\hline Sfla_4041 & N/A & regulatory protein \\
\hline Sfla_4131 & pfam04149 & BldB family \\
\hline Sfla_4132 & pfam01381 & $\mathrm{HBP}$ \\
\hline Sfla_4158 & N/A & hypothetical \\
\hline Sfla_4267 & N/A & hypothetical \\
\hline
\end{tabular}

Table 3 Highly divergent core genes (Continued)

\begin{tabular}{|c|c|c|}
\hline Sfla_4283 & pfam00535 & glycosyl transferase family 2 \\
\hline Sfla_4288 & cd03786 & $\begin{array}{l}\text { UDP-N-Acetylglucosamine } \\
\text { (GlcNAc) 2-Epimerase }\end{array}$ \\
\hline Sfla_4402 & N/A & Fasciclin-like protein \\
\hline Sfla_4439 & pfam04149 & BldB family \\
\hline Sfla_4440 & N/A & HBP \\
\hline Sfla_4606 & pfam04149 & BldB family \\
\hline Sfla_4607 & N/A & HBP \\
\hline Sfla_4660 & COG0841 & Cation/multidrug effluN/A pump \\
\hline Sfla_5089 & COG0641 & Arylsulfatase regulator \\
\hline Sfla_5090 & N/A & hypothetical \\
\hline Sfla_5091 & pfam00931 & NB-ARC \\
\hline Sfla_5365 & pfam02618 & YceG-like family \\
\hline Sfla_5366 & PRK00258 & shikimate 5-dehydrogenase \\
\hline Sfla_6367 & N/A & hypothetical \\
\hline Sfla_6381 & pfam00440 & tetR family regulator \\
\hline Sfla_6389 & N/A & hypothetical \\
\hline
\end{tabular}

A total of 59 core genes were found to have unusually high sequence variability ( $>5 \%$ nucleotide dissimilarity) between strains. These genes are listed using the IAF-45-CD sequence annotation and classified using CDD and RPSBLAST.

contains two copies of $b l d B$ and only one copy of $h p b$ (highlighted in blue in Figure 5 and Figure 6). The gene phylogenies are consistent with a general pattern of coevolution with new gene pairs arising due to duplication, although lateral gene transfer has clearly affected the phylogeny (Figure 5). There is also a consistent relationship between the frequency of substitutions in the BldB orthologs and their $\mathrm{Hpb}$ partners (Figure 6).

\section{Effect of homologous recombination}

SNP analysis revealed widespread recombination across the genome. Informative sites, which are sites at which two genomes share a SNP (i.e. it is not present in only one genome), can be evaluated with the four-gamete test [44] to identify polymorphisms acquired through recombination. The mean distance between incompatible sites is 1,191 bases indicating that each genome is a mosaic of intraspecies recombination. Informative SNPs shared by two strains support all possible patterns of ancestry among these five strains (Table 4) and this indicates that frequent recombination has erased the intraspecies phylogenetic signal across the genome. If this population were clonal, then informative SNPs would support a bifurcating pattern of ancestry with informative SNPs present only in strains that share a unique common ancestor to the exclusion of other genomes in the analysis.

To determine the effect of recombination on phylogenetic reconstruction within this species, we determined the "phylogenetic signal" across the genome; a value of 1 


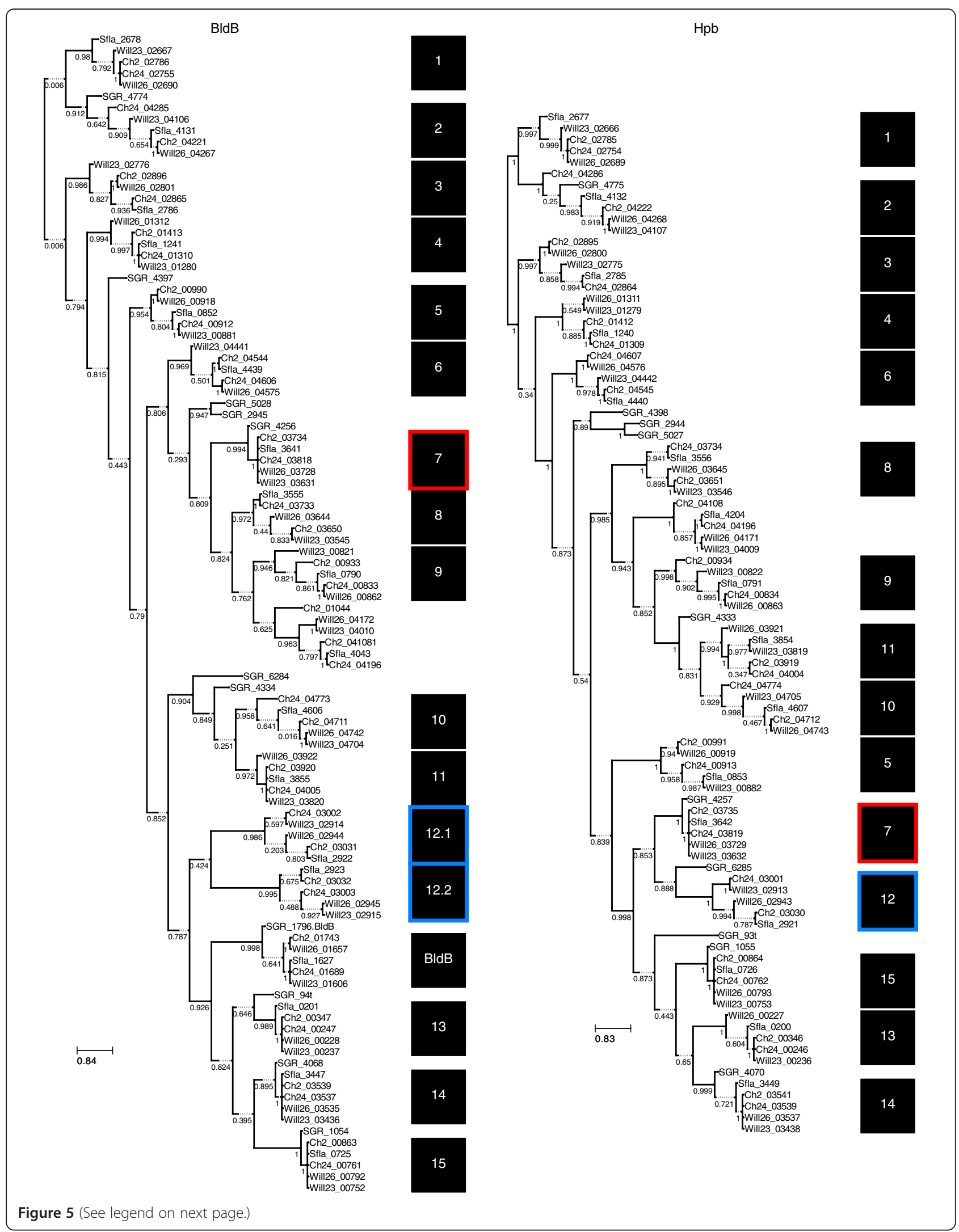


(See figure on previous page.)

Figure 5 BldB and Hpb phylogenies. Maximum likelihood trees constructed from amino acid sequences of BldB homologs (left) and $\mathrm{Hpb}$ homologs (right). Sequence names indicate strain identity via locus tags. Values on edges represent SH-based local support values. The branch length represents evolutionary distance using the Gamma20 model within FastTree 2 [38]. Additional length is given to some branches with dashed lines to allow room for bootstrap values. Adjacent bldB and $h p b$ genes are indicated in the two trees by numbered boxes to the right of the trees. Not all occurrences of bldB or $\mathrm{hpb}$ are accompanied by the other gene, as is the case with bldB originally characterized from Streptomyces griseus NBRC 13350 (SGR_1796.BldB). Box 7, highlighted in red, indicates incongruence between bldB and corresponding hpb gene phylogeny suggesting horizontal acquisition or gene conversion at one of these loci. Boxes 12.1 and 12.2, highlighted in blue, show an instance where bldB was duplicated locally without accompanying duplication of $h p b$.

indicates clonal inheritance, 0 indicates a random assortment of sites due to homologous recombination. For every informative SNP, the distance and compatibility of every other informative SNPs within $20 \mathrm{~kb}$ is used to calculate phylogenetic signal after combining the values into $100 \mathrm{bp}$ bins. The phylogenetic signal in the $S$. pratensis genomes declined exponentially with distance (Figure 7). The nonlinear regression model fit to the data (implemented in $\mathrm{R}$ ) is $y=e^{(-0.0002071 x)}$ where $y$ is the phylogenetic signal and $x$ is chromosomal distance. For comparison, the same analysis was performed on five genomes from the East Asian population of Helicobacter pylori (Figure 7), which is known to be highly recombinogenic [45]. Likewise, five genomes of Mycobacterium tuberculosis, a clonal species with low rates of recombination [46], were similarly analyzed (Figure 7). This result shows that the phylogeny

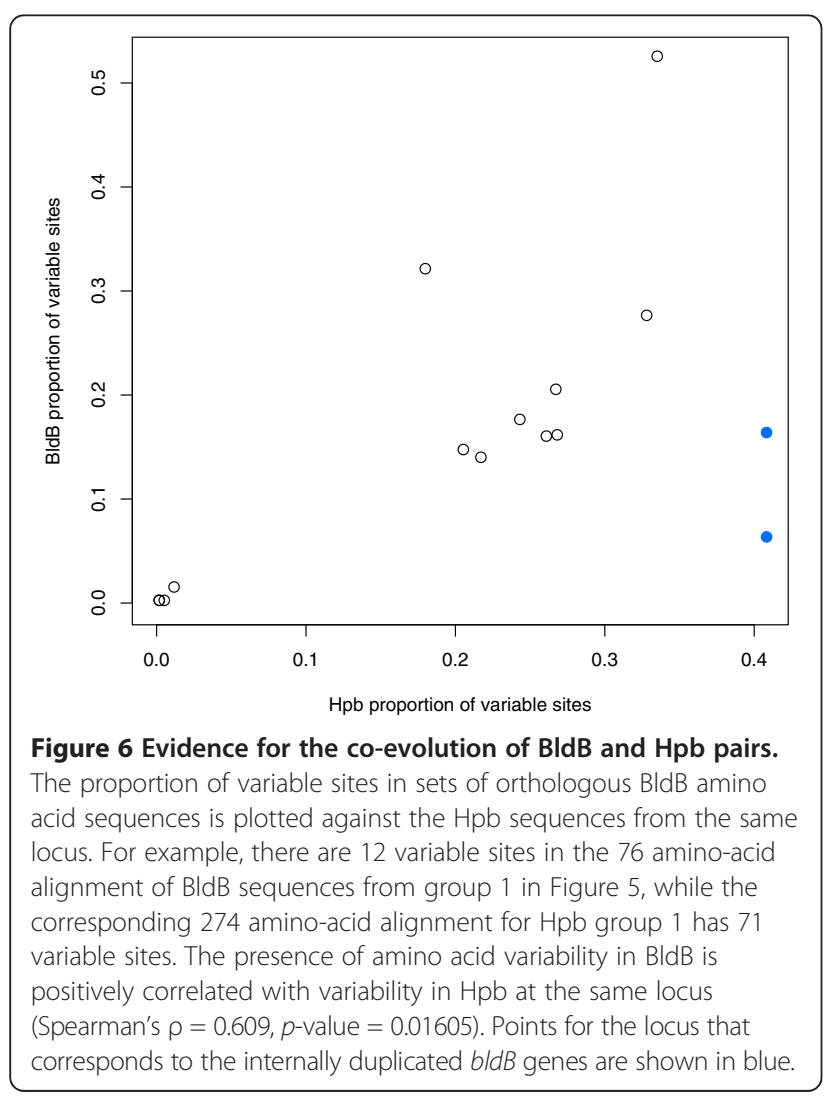

supported by an informative site within $S$. pratensis has no bearing on the phylogeny of a site $\geq 15-20 \mathrm{~kb}$ distant.

The recombination analysis revealed an anomalous region of the genome that lacked incompatible sites over a span of 20,134 bp. The length of this region is unlikely to occur due to chance $(p<0.01)$ based on the background rate of intraspecies recombination in the genome (Figure 7). This region was centered on an indel affecting genes Sfla_5857 and Sfla_5858 (Figure 8). These genes are annotated as a MarR-like regulator and a hypothetical protein, respectively. The insertion affects the terminal end of Sfla_5858 and the start of Sfla_5857. Also included in this region is a natural product biosynthetic gene cluster which comprises a type III polyketide synthase, a lycopene cyclase, a prenyltransferase (UbiA) and an AurF-like N-oxygenase (Figure 8). AurF is responsible for formation of the nitro- group in aureothin biosynthesis [47]. The indel mutation discriminates Ch24, Will26, and Will23 from Ch2 and IAF-45-CD (Figure 8). Linkage equilibrium has broken down around this indel with local patterns of recombination between genomes defined by the presence or absence of the indel mutation. The cause of linkage disequilibrium is unknown and could result from either a partial selective sweep enabled by horizontal transfer of this genomic island, selection against recombinants driven by the presence of the indel mutation in this region of the genome, or some unknown molecular mechanism which may be inhibiting recombination locally in response to the indel mutation.

A second genomic island is also found to lack incompatible sites, and the size of this region is unexpected to result from chance $(p<0.01)$ based on the background rate of intraspecies recombination in the genome (Figure 7).

Table 4 Analysis of informative SNPs and variable genes

\begin{tabular}{lccccc}
\hline & Sfla & Will23 & Will26 & Ch2 & Ch24 \\
\hline Sfla & - & 153 & 187 & 149 & 148 \\
Will23 & 3334 & - & 303 & 323 & 361 \\
Will26 & 4467 & 2947 & - & 291 & 274 \\
Ch2 & 3865 & 2510 & 2538 & - & 360 \\
Ch24 & 3367 & 2773 & 3317 & 2182 & - \\
\hline
\end{tabular}

Pairwise analysis of informative SNPs are shown below the diagonal and shared variable genes present in only two genomes are shown above. 


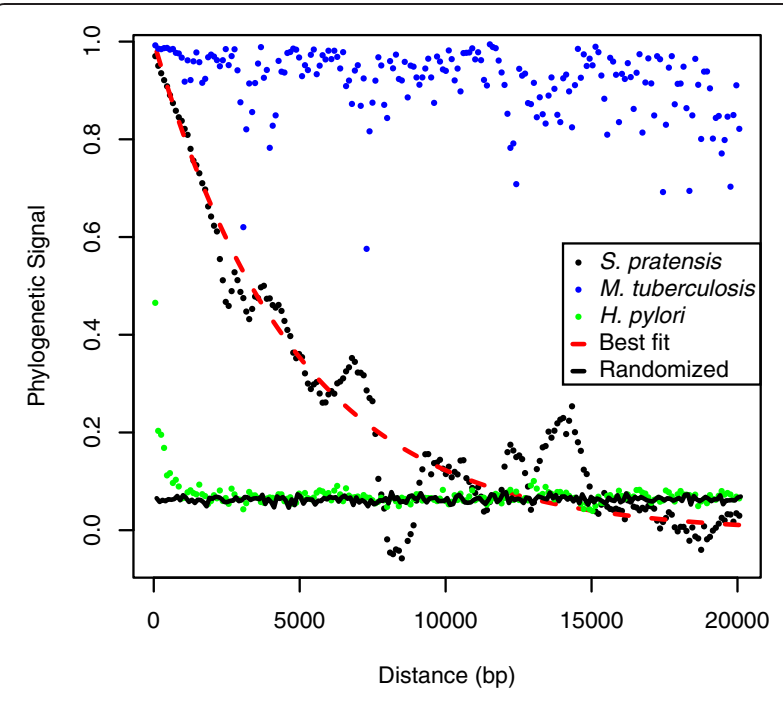

Figure 7 Recombination erases the intraspecies phylogenetic signal in S. pratensis. The proportion of incompatible sites along the five S. pratensis chromosomes is a function of chromosome distance. Phylogenetic signal is defined by a value of 1 indicating an absence of incompatible sites (pure vertical pattern of inheritance) and a value of 0 indicating that incompatible sites are randomly distributed by recombination. The solid black line indicates a random equilibrium SNP distribution generated from shuffling all SNPs among the $S$. pratensis genomes. Results are shown for the analysis of five $S$. pratensis genomes (black circles), as well as five Mycobacterium tuberculosis genomes which are known to represent a highly clonal species (blue circles) and five Helicobacter pylori genomes which are known to represent a highly recombining population (green circles). An exponential decay is fitted to the $S$. pratensis decay in phylogenetic signal due to distance between sites (dashed red line).

The region occurs within a hybrid NRPS-PKS biosynthetic gene cluster (Sfla_6220-1) and spans 15696 bp. In this region the genomes IAF- $45-\mathrm{CD}$ and $\mathrm{Ch} 2$ do not have any evidence of recombination with the genomes Will23, Will26 and Ch24. This is the same pattern that is seen in the region of Sfla_5857-8 as discussed previously. The IAF-45-CD genome contains a single base insertion within this region that introduces a stop codon in a ketosynthase domain.

\section{Discussion}

The size of the core genome can vary widely between bacterial species. For example, core genes can represent anywhere from $20 \%$ to $93 \%$ of the total genome across diverse bacterial lineages such as: Escherichia coli, 20\% [48]; Streptococcus pneumoniae, 52\% [49]; Salmonella enterica, 61\% [50]; Actinobacillus pleuropneumoniae, 79\% [51]; Listeria monocytogenes, 80\% [52]; Campylobacter coli, 82.4\% [53]; Campylobacter jejuni, 83.5\% [53]; and Chlamydia trachomatis, 93\% [54]. We have used a very strict definition of a core genome in our analysis of S. pratensis, using only positionally orthologous genes, as opposed to gene families, unlike many of the studies listed. Even with this strict definition, we calculate that $85-88 \%$ of each genome is comprised of core genes. Because our metric is more stringent than bi-directional best BLAST hits, which is used in many publications, these estimates should be viewed as a conservative estimate in comparison. Since the vast majority of variable genes are unique to a single genome (Figures 1A) and the estimate of core genome size changed little between the second and fifth genomes added to the analysis (Figure 1B) it is unlikely that the addition of new genomes will reduce substantially the size of the core genome in S. pratensis. Among published bacterial genome comparisons, only Chlamydia trachomatis, an obligate intracellular pathogen that has experienced extreme genome reduction and is left with only $~ 900$ genes in the average genome [54], has a larger core genome proportion than S. pratensis. In addition, our assessment of the core genome does not exclude genes of putative plasmid origin, and as such represents a lower bound of core genome content for the linear chromosome. These observations suggest that the genome of $S$. pratensis is overwhelmingly dominated by core genes.

Comparative genomics of different species of Streptomyces has indicated that the central portion of the chromosome is highly conserved both in gene content and synteny while chromosome termini are highly variable between species. Our intraspecies comparison reveals islands of diversity spread throughout the chromosome, including at the terminal variable regions but also throughout the central core. The terminal variable regions are only 112 $\mathrm{kbp}$ (the $5^{\prime}$ arm as annotated in ATCC 33331) and $36 \mathrm{kbp}$ (Figure 1D). This is significantly smaller than the 753 to 1,393 kbp terminal arms found in interspecific comparisons [55]. Strain specific islands are composed primarily of mobile genetic elements and genes that are likely to have been acquired by horizontal gene transfer. Strain specific islands composed of mobile genetic elements are a common feature of many microbial genomes.

All five of these genomes have the same repertoire of natural product gene clusters which are unambiguously part of the core genome of $S$. pratensis. This means that efforts to mine genomes for novel gene clusters will be facilitated by accurate species classifications, which can eliminate the need to needlessly screen many strains of the same species. However, our results for the bldB and $h p b$ gene families suggest that changes to regulatory genes can occur at a fast rate within a species and may affect the expression of gene clusters between strains of a species. Examining diverse isolates from the same species may allow researchers to find regulatory changes that activate gene clusters that are otherwise cryptic in other strains. We have also shown that lanthipeptide precursors and NRPS and PKS genes can be highly variable within a species. 


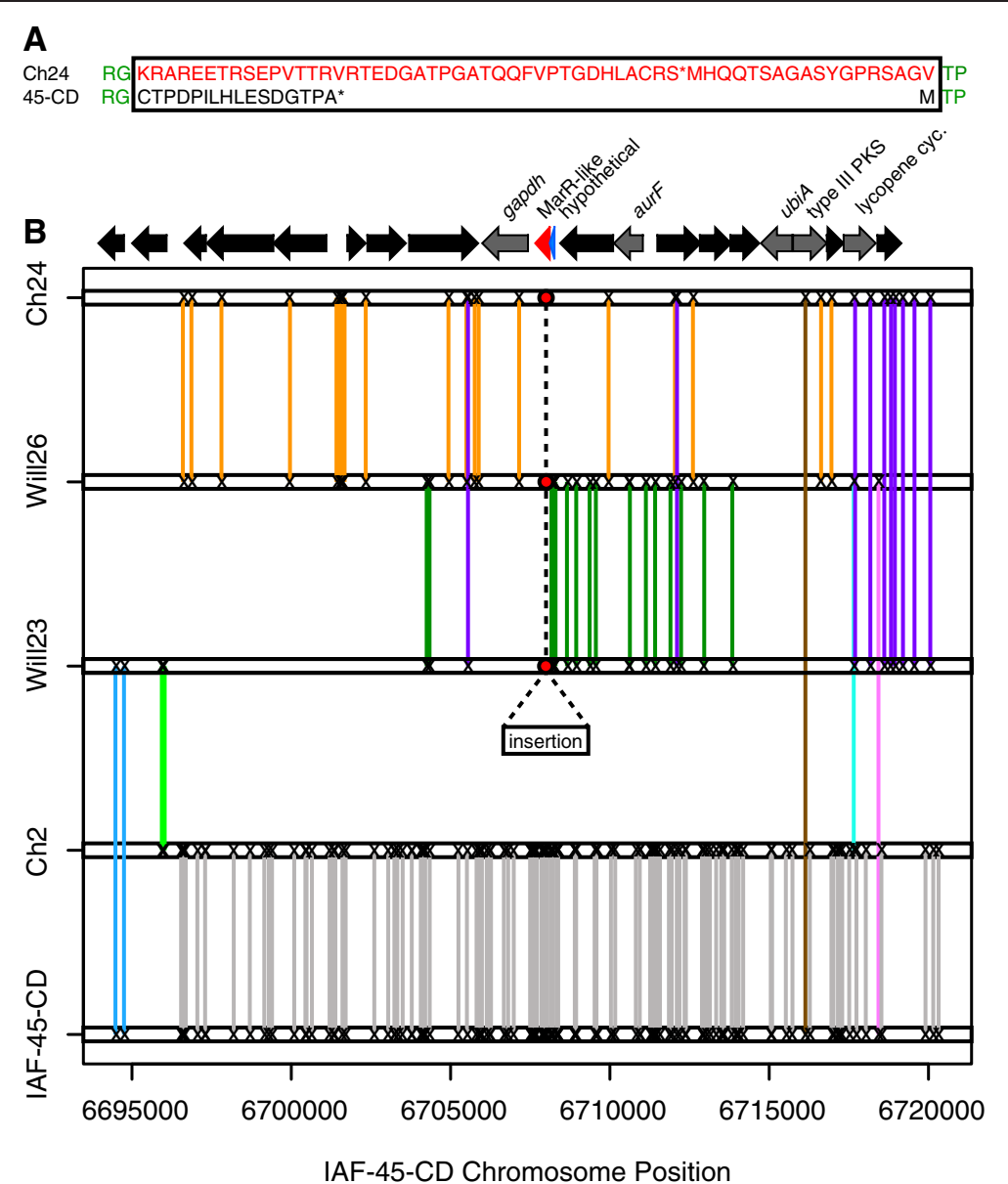

Figure 8 Linkage region. The S. pratensis genomes contain a 20 kbp region in which linkage equilibrium has broken down, suggesting a decrease in recombination between the genomes of Ch24, Will26, Will23 and those of Ch2, IAF-45-CD. The predicted amino acid sequences affected by an indel at the center of this region is shown in panel $\mathbf{A}$. The amino acid sequences of Will26 and Will23 are identical in this region and differ from Ch24 by only one residue (only Ch24 is shown in panel A).The affected amino acid sequence is surrounded by a box. The location of the indel is indicated by a rectangle and red dots in panel B. Informative SNPs are indicated on each genome segment by an ' $X$ ' and colored lines indicate pairs of genomes which share each SNP. Stop codons are indicated with an asterisk in panel $\mathbf{A}$.

S. pratensis possesses a new combination of putative beta-lactam (MM 4550-like) and putative beta-lactamase inhibitor (clavulanic acid-like) biosynthetic gene clusters. This observation, on one level, suggests that observing patterns of natural product gene-clusters within the genomes of actinomycetes may reveal new possible drug combinations that have been proven effective by the crucible of evolution, and such new combinations may prove useful therapeutically. This observation also provides evidence for the in situ use of these products as antibiotics rather than as signaling molecules [56]. While the production of these molecules in S. pratensis has not yet been verified experimentally, the observation that convergent evolution has produced divergent combinations of beta-lactam and beta-lactamase inhibitor gene clusters independently in both $S$. pratensis and S. clavuligerus (species that do not share a common ancestor within Streptomyces) is evidence for the presence of an evolutionary arms race in soil communities. That is, selection has on at least two occasions driven the independent assembly of systems designed to produce both beta-lactam antibiotics and overcome beta-lactam resistance. The selection pressure for this pattern of gene cluster co-occurrence can only be explained by the hypothesis that: i) there is a high frequency of beta-lactam resistance in soils, and ii) these streptomyces benefit from the use of beta-lactam antibiotics to inhibit or kill other microorganisms in the soil community.

We have found that there is no consensus phylogenetic signal among $S$. pratensis genomes; the genome represents a mosaic of recombination between strains of the species. In this way $S$. pratensis resembles the East Asian $H$. pylori population in that recombination scrambles patterns of polymorphism between strains. A difference between these two species is that phylogenetic signal decays over shorter distances in $H$. pylori than in 
S. pratensis (Figure 7 ). This result could be caused by differences both in recombination rate and in the tract length of recombination. For example, H. pylori is naturally competent and can incorporate short stretches of DNA into its genome through transformation [45]. In contrast, acquisition of DNA by Streptomyces proceeds through an unusual dsDNA dependent mechanism of conjugation that may result in transfer of the whole chromosomes and backcrossing with the parent [7].

The frequency of recombination in S. pratensis was such that there is no detectable association between geographic distance and genomic divergence between strains from NY and NC. The geographic range of S. pratensis is unknown and it is not possible to estimate rates of migration from our current sample size, or to estimate whether dispersal limitation impacts the biogeographic pattern of genetic diversity within the species at very large spatial scales (e.g. continental). What we have shown, however, is that the extent of recombination between stains isolated from soils $740 \mathrm{~km}$ apart is not discernably different from the recombination observed between strains that co-occur in the same soil sample. Such a pattern could result from contemporaneous gene exchange at regional spatial scales (i.e. recombination between sites exceeds the ability of mutation to promote divergence between sites). Alternatively, this pattern could also result from the evolutionary recent regional expansion of a population that was recently in equilibrium.

While the majority of positionally orthologous core genes were highly similar between genomes, we observed 59 positional orthologs that differed by more than $5 \%$ between the $S$. pratensis genomes. Of these, 19 are members of two gene families known to be involved in regulation of differentiation and development. These two co-occurring gene families are BldB and what we have termed $\mathrm{Hpb}$ (for helix-turn-helix partner of BldB). BldB is a transcriptional regulator previously shown to be required for development of aerial mycelia, e.g. bldB mutants have bald colonies [57]. The conserved domain found within BldB is in the conserved domain database as DUF397 [34]. OrfD (SCO0703) in the antibiotic regulatory locus $a b a A$ and the developmental regulator WhiJ also fall within this class [58]. BldB has been characterized as a DNA-binding repressor that down-regulates its own expression [59], and was also previously suggested to have a binding partner that modulates BldB activity [60]. Hpb is predicted to have a helix-turn-helix domain of the Xre class. Based on the presence of a Xre domain, a history of gene duplication and recent proliferation, and limitation to a subset of the Actinobacteria, this gene pair was predicted by Makarova et al. [61] to represent a novel toxin-antitoxin system. The correspondence in diversification of the $b l d b / h p b$ family gene pairs suggests that a mutation in one gene encourages a compensatory change in its partner at the same locus. These observations from $S$. pratensis provide further indirect evidence that BldB-Hpb functions in a manner that resembles a toxin-antitoxin system.

\section{Conclusion}

We have uncovered patterns of genome evolution within a Streptomyces species through comparison of S. pratensis genomes isolated from disparate geographic origins. Core genes make up a high percentage of the genome, and natural product genes are unambiguously included within the set of core genes. High rates of intraspecies recombination homogenize polymorphisms in core genes across the genome and abolish any phylogenetic signal present within species. Two genomic islands exhibited a breakdown in intraspecies recombination promoting localized divergence between sets of genomes. One of these islands was centered on a hybrid NRPS-PKS gene, and the other was centered on a regulatory gene associated with a type III polyketide synthase biosynthetic gene cluster. In addition, highly divergent core genes included a lanthipeptide biosynthetic gene cluster as well as co-occurring members of the bldB and $h p b$ gene families, genes which have been associated with the regulation of development and antibiotic production in Streptomyces. The conservation of biosynthetic gene clusters between strains of the species suggests that antibiotic production capacity is conserved within a species while variation in $b l d B$ and $h p b$ gene families suggest that changes to regulatory genes can occur at a fast rate within a species and may affect the expression of biosynthetic gene clusters between strains of a species.

\section{Competing interests}

The authors declare that they have no competing interests.

\section{Authors' contributions}

JRD and DHB designed the work and wrote the manuscript. JRD performed the work and DHB guided the work. Both authors read and approved the final manuscript.

\section{Acknowledgements}

JRD was funded by a Cornell Center for Comparative and Population Genomics Graduate Student Fellowship while at Cornell University and an Institute for Genomic Biology Postdoctoral Fellowship while at the University of Illinois at Urbana-Champaign. Genome sequencing was performed with funds provided by the Cornell Center for Comparative and Population Genomics. This material is based in part upon work supported by the National Science Foundation under Grant No. (DEB-1050475).

\section{Author details}

${ }^{1}$ Institute for Genomic Biology, University of Illinois at Urbana-Champaign, Urbana, USA. ${ }^{2}$ Department of Crop and Soil Sciences, Cornell University, Ithaca, USA.

Received: 26 June 2014 Accepted: 29 October 2014

Published: 15 November 2014

\section{References}

1. Bérdy J: Bioactive microbial metabolites. J Antibiot (Tokyo) 2005, 58(1):1-26.

2. Rong $X$, Liu N, Ruan J, Huang $Y$ : Multilocus sequence analysis of

Streptomyces griseus isolates delineating intraspecific diversity in terms of both taxonomy and biosynthetic potential. Antonie Van Leeuwenhoek 2010, 98(2):237-248. 
3. Rong $X Y$, Huang Y: Taxonomic evaluation of the Streptomyces griseus clade using multilocus sequence analysis and DNA-DNA hybridization, with proposal to combine 29 species and three subspecies as 11 genomic species. Int J Syst Evol Microbiol 2010, 60:696-703.

4. Guo Y, Zheng W, Rong X, Huang Y: A multilocus phylogeny of the Streptomyces griseus $16 \mathrm{~S}$ rRNA gene clade: use of multilocus sequence analysis for streptomycete systematics. Int J Syst Evol Microbiol 2008, 58(1):149-159.

5. Rong $X Y$, Huang Y: Taxonomic evaluation of the Streptomyces hygroscopicus clade using multilocus sequence analysis and DNA-DNA hybridization, validating the MLSA scheme for systematics of the whole genus. Syst Appl Microbiol 2012, 35(1):7-18.

6. Rong XY, Guo YP, Huang Y: Proposal to reclassify the Streptomyces albidoflavus clade on the basis of multilocus sequence analysis and DNA-DNA hybridization, and taxonomic elucidation of Streptomyces griseus subsp solvifaciens. Syst Appl Microbiol 2009, 32(5):314-322.

7. Hopwood DA: Soil to genomics: the streptomyces chromosome. Annu Rev Genet 2006, 40:1-23.

8. Doroghazi JR, Buckley DH: Widespread homologous recombination within and between Streptomyces species. ISME J 2010, 4(9):1136-1143.

9. Flardh K: Essential role of DivIVA in polar growth and morphogenesis in Streptomyces coelicolor A3(2). Mol Microbiol 2003, 49(6):1523-1536.

10. Schwedock J, McCormick JR, Angert ER, Nodwell JR, Losick R: Assembly of the cell division protein FtsZ into ladder-like structures in the aerial hyphae of Streptomyces coelicolor. Mol Microbiol 1997, 25(5):847-858.

11. Hopwood DA, Glauert AM: Observations on the chromatinic bodies of Streptomyces coelicolor. J Biophys Biochem Cytol 1960, 8(1):257-265.

12. Lin YS, Kieser HM, Hopwood DA, Chen CW: The chromosomal DNA of Streptomyces lividans 66 is linear. Mol Microbiol 1993, 10(5):923-933.

13. Lin YS, Chen CW: Instability of artificially circularized chromosomes of Streptomyces lividans. Mol Microbiol 1997, 26(4):709-719.

14. Volff JN, Viell P, Altenbuchner J: Artificial circularization of the chromosome with concomitant deletion of its terminal inverted repeats enhances genetic instability and genome rearrangement in Streptomyces lividans. Mol Gen Genet 1997, 253(6):753-760.

15. Weaver D, Karoonuthaisiri N, Tsai HH, Huang CH, Ho ML, Gai SN, Patel KG, Huang JQ, Cohen SN, Hopwood DA, Chen CW, Kao CM: Genome plasticity in Streptomyces: identification of $1 \mathrm{Mb}$ TIRs in the S. coelicolor A3(2) chromosome. Mol Microbiol 2004, 51(6):1535-1550.

16. Ikeda H, Ishikawa J, Hanamoto A, Shinose M, Kikuchi H, Shiba T, Sakaki Y, Hattori M, Ōmura S: Complete genome sequence and comparative analysis of the industrial microorganism Streptomyces avermitilis. Nat Biotechnol 2003, 21(5):526-531.

17. Hopwood DA, Wright HM: A plasmid of Streptomyces coelicolor carrying a chromosomal locus and its inter-specific transfer. The J Gen Microbiol 1973, 79(2):331-342.

18. Ohnishi $Y$, Ishikawa J, Hara H, Suzuki H, Ikenoya M, Ikeda H, Yamashita A, Hattori M, Horinouchi S: Genome sequence of the streptomycinproducing microorganism streptomyces griseus IFO 13350. J Bacteriol 2008, 190(11):4050-4060

19. Bentley SD, Chater KF, Cerdeno-Tarraga AM, Challis GL, Thomson NR, James KD, Harris DE, Quail MA, Kieser H, Harper D, Bateman A, Brown S, Chandra G, Chen CW, Collins M, Cronin A, Fraser A, Goble A, Hidalgo J, Hornsby T, Howarth S, Huang CH, Kieser T, Larke L, Murphy L, Oliver K, O'Neil S, Rabbinowitsch E, Rajandream MA, Rutherford Ket al: Complete genome sequence of the model

actinomycete Streptomyces coelicolor A3(2). Nature 2002, 417(6885):141-147.

20. Omura S, Ikeda H, Ishikawa J, Hanamoto A, Takahashi C, Shinose M, Takahashi Y, Horikawa H, Nakazawa H, Osonoe T, Kikuchi H, Shiba T, Sakaki $Y$, Hattori M: Genome sequence of an industrial microorganism Streptomyces avermitilis: deducing the ability of producing secondary metabolites. Proc Natl Acad Sci U S A 2001, 98(21):12215-12235.

21. Kharel MK, Nybo SE, Shepherd MD, Rohr J: Cloning and characterization of the ravidomycin and chrysomycin biosynthetic gene clusters. Chembiochem 2010, 11(4):523-532.

22. Ziemert N, Lechner A, Wietz M, Millán-Aguiñaga N, Chavarria KL, Jensen PR: Diversity and evolution of secondary metabolism in the marine actinomycete genus Salinispora. Proc Natl Acad Sci U S A 2014, 111(12):E1130-E1139.

23. Rong $X$, Doroghazi JR, Cheng $K$, Zhang L, Buckley DH, Huang Y: Classification of Streptomyces phylogroup pratensis (Doroghazi and Buckley, 2010) based on genetic and phenotypic evidence, and proposal of Streptomyces pratensis sp. nov. Syst Appl Microbiol 2013, 36(6):401-407.
24. Doroghazi JR, Buckley DH: A model for the effect of homologous recombination on microbial diversification. Genome Biol Evol 2011, 3:1349.

25. Fraser C, Hanage WP, Spratt BG: Recombination and the nature of bacterial speciation. Science 2007, 315(5811):476-480.

26. Ishaque M, Kluepfel D: Cellulase complex of a mesophilic Streptomyces strain. Can J Microbiol 1980, 26(2):183-189.

27. El-Nakeeb MA, Lechevalier HA: Selective isolation of aerobic actinomycetes. Appl Microbiol 1963, 11(2):75-77.

28. Ottow JCG: Rose Bengal as a selective aid in the isolation of fungi and actinomycetes from natural sources. Mycologia 1972, 64(2):304-315.

29. Darling ACE, Mau B, Blattner FR, Perna NT: Mauve: multiple alignment of conserved genomic sequence with rearrangements. Genome Res 2004 14(7):1394-1403.

30. Hyatt D, Chen GL, LoCascio PF, Land ML, Larimer FW, Hauser LJ: Prodigal: prokaryotic gene recognition and translation initiation site identification. BMC Bioinform 2010, 11:119.

31. Larkin MA, Blackshields G, Brown NP, Chenna R, McGettigan PA, McWilliam $H$, Valentin F, Wallace IM, Wilm A, Lopez R, Thompson JD, Gibson TJ, Higgins DG: Clustal W and clustal X version 2.0. Bioinformatics 2007, 23(21):2947-2948.

32. Felsenstein J: Confidence limits on phylogenies: an approach using the bootstrap. Evolution 1985, 39(4):783-791.

33. Altschul SF, Madden TL, Schaffer AA, Zhang JH, Zhang Z, Miller W, Lipman DJ: Gapped BLAST and PSI-BLAST: a new generation of protein database search programs. Nucleic Acids Res 1997, 25(17):3389-3402.

34. Marchler-Bauer A, Lu S, Anderson JB, Chitsaz F, Derbyshire MK, DeWeese-Scott C, Fong JH, Geer LY, Geer RC, Gonzales NR, Gwadz M, Hurwitz DI, Jackson JD, Ke Z, Lanczycki CJ, Lu F, Marchler GH, Mullokandov M, Omelchenko MV, Robertson CL, Song JS, Thanki N, Yamashita RA, Zhang D, Zhang N, Zheng C, Bryant SH: CDD: a conserved domain database for the functional annotation of proteins. Nucleic Acids Res 2011, 39:D225-D229.

35. Alexa A, Rahnenfuhrer J: topGO: topGO: Enrichment analysis for Gene Ontology. R Package Version 2.18.0 2010, 28.

36. Gentleman RC, Carey VJ, Bates DM, Bolstad B, Dettling M, Dudoit S, Ellis B, Gautier L, Ge Y, Gentry J: Bioconductor: open software development for computational biology and bioinformatics. Genome Biol 2004, 5(10):R80.

37. Medema MH, Blin K, Cimermancic $P$, de Jager $V$, Zakrzewski $P$, Fischbach MA, Weber T, Takano E, Breitling R: AntiSMASH: rapid identification, annotation and analysis of secondary metabolite biosynthesis gene clusters in bacterial and fungal genome sequences. Nucleic Acids Res 2011, 39(suppl 2):W339-W346.

38. Price MN, Dehal PS, Arkin AP: FastTree 2-approximately maximum-likelihood trees for large alignments. PLoS One 2010, 5(3):e9490.

39. Huerta-Cepas J, Dopazo J, Gabaldón T: ETE: a python Environment for Tree Exploration. BMC Bioinform 2010, 11(1):24.

40. Li R, Lloyd EP, Moshos KA, Townsend CA: Identification and characterization of the carbapenem MM 4550 and its gene cluster in streptomyces argenteolus ATCC 11009. Chem Bio Chem 2014, 15(2):320-331.

41. Nunez LE, Mendez C, Brana AF, Blanco G, Salas JA: The biosynthetic gene cluster for the beta-lactam carbapenem thienamycin in Streptomyces cattleya. Chem Biol 2003, 10(4):301-311.

42. Doroghazi JR, Albright JC, Goering AW, Ju K-S, Haines RR, Tchalukov KA, Labeda DP, Kelleher NL, Metcalf WW: A roadmap for natural product discovery based on large-scale genomics and metabolomics. Nat Chem Biol 2014, 10(11):963-968.

43. Boratyn GM, Schaffer A, Agarwala R, Altschul SF, Lipman DJ, Madden TL: Domain enhanced lookup time accelerated BLAST. Biol Direct 2012, 7(1):12.

44. Hudson R, Kaplan N: Statistical properties of the number of recombination events in the history of a sample of DNA sequences. Genetics 1985, 111(1):147-211

45. Suerbaum S, Smith JM, Bapumia K, Morelli G, Smith NH, Kunstmann E, Dyrek I, Achtman M: Free recombination within Helicobacter pylori. Proc Nat Acad Sci U S A 1998, 95(21):12619-12624.

46. Supply P, Warren RM, Bañuls AL, Lesjean S, Van Der Spuy GD, Lewis LA, Tibayrenc M, Van Helden PD, Locht C: Linkage disequilibrium between minisatellite loci supports clonal evolution of Mycobacterium tuberculosis in a high tuberculosis incidence area. Mol Microbiol 2003, 47(2):529-538.

47. He J, Hertweck C: Biosynthetic origin of the rare nitroaryl moiety of the polyketide antibiotic aureothin: involvement of an unprecedented N-oxygenase. J Am Chem Soc 2004, 126(12):3694-3695. 
48. Lukjancenko O, Wassenaar TM, Ussery DW: Comparison of 61 sequenced escherichia coli genomes. Microb Ecol 2010, 60(4):708-720.

49. Donati C, Hiller NL, Tettelin H, Muzzi A, Croucher NJ, Angiuoli SV, Oggioni M, Hotopp JCD, Hu FZ, Riley DR, Covacci A, Mitchell TJ, Bentley SD, Kilian M, Ehrlich GD, Rappuoli R, Moxon ER, Masignani V: Structure and dynamics of the pan-genome of Streptococcus pneumoniae and closely related species. Genome Biol 2010, 11(10):R107.

50. Jacobsen A, Hendriksen RS, Aaresturp FM, Ussery DW, Friis C: The Salmonella enterica Pan-genome. Microb Ecol 2011, 62(3):487-504.

51. Xu Z, Chen X, Li L, Li T, Wang S, Chen H, Zhou R: Comparative genomic characterization of actinobacillus pleuropneumoniae. J Bacteriol 2010, 192(21):5625-5636.

52. Deng X, Phillippy AM, Li Z, Salzberg SL, Zhang W: Probing the pangenome of Listeria monocytogenes: new insights into intraspecific niche expansion and genomic diversification. BMC Genomics 2010, 11(1):500.

53. Lefebure T, Bitar PDP, Suzuki H, Stanhope MJ: Evolutionary dynamics of complete campylobacter Pan-genomes and the bacterial species concept. Genome Biol Evol 2010, 2:646-655.

54. Joseph SJ, Didelot X, Gandhi K, Dean D, Read TD: Interplay of recombination and selection in the genomes of Chlamydia trachomatis. Biol Direct 2011, 6:28.

55. Choulet F, Aigle B, Gallois A, Mangenot S, Gerbaud C, Truong C, Francou F-X, Fourrier $C$, Guérineau M, Decaris B: Evolution of the terminal regions of the Streptomyces linear chromosome. Mol Biol Evol 2006, 23(12):2361-2369.

56. Yim G, Wang HH, FRS JD: Antibiotics as signalling molecules. Philos Transact Royal Society B: Biol Sci 2007, 362(1483):1195-1200.

57. Champness WC: New loci required for Streptomyces coelicolor morphological and physiological differentiation. J Bacterio/ 1988, 170(3):1168-1174.

58. Eccleston M, Ali RA, Seyler R, Westpheling J, Nodwell J: Structural and genetic analysis of the BldB protein of streptomyces coelicolor. J Bacteriol 2002, 184(15):4270-4276.

59. Pope MK, Green B, Westpheling J: The bldB gene encodes a small protein required for morphogenesis, antibiotic production, and catabolite control in Streptomyces coelicolor. J Bacteriol 1998, 180(6):1556-1562.

60. Eccleston M, Willems A, Beveridge A, Nodwell JR: Critical residues and novel effects of overexpression of the streptomyces coelicolor developmental protein BldB: evidence for a critical interacting partner. J Bacteriol 2006, 188(23):8189-8195.

61. Makarova KS, Wolf Yl, Koonin EV: Comprehensive comparative-genomic analysis of type 2 toxin-antitoxin systems and related mobile stress response systems in prokaryotes. Biol Direct 2009, 4(1):19.

doi:10.1186/1471-2164-15-970

Cite this article as: Doroghazi and Buckley: Intraspecies comparison of Streptomyces pratensis genomes reveals high levels of recombination and gene conservation between strains of disparate geographic origin BMC Genomics 2014 15:970.

\section{Submit your next manuscript to BioMed Central and take full advantage of:}

- Convenient online submission

- Thorough peer review

- No space constraints or color figure charges

- Immediate publication on acceptance

- Inclusion in PubMed, CAS, Scopus and Google Scholar

- Research which is freely available for redistribution 